\title{
El Programa ACTA (Administración, Control y Tratamiento de Archivos) y el Archivo Central del Servicio Andaluz de Salud
}

Reyes Pro Jiménez

Directora Archivo Central del Servicio Andaluz de Salud

\section{Resumen}

El desarrollo e implementación del programa ACTA de gestión de archivos administrativos ha sido fundamental para garantizar la accesibilidad a los datos contenidos en toda clase de documentos del SAS.

La filosofía que sigue el programa es la sencillez de uso y presentación, y la inclusión de todas las tareas de gestión de archivo y recuperación de la documentación administrativa.

El programa es además un ejemplo de colaboración entre distintos campos de las ciencias de la Documentación e Información, ya que el método de trabajo seguido se ha basado en una estrecha colaboración entre informáticos, archiveros y documentalistas, aportando cada uno las técnicas propias.

El programa ACTA tiene tres módulos: administrador, usuario de archivo y oficina, en ellos tienen una importancia primordial el análisis funcional de la organización, el establecimiento de una clasificación documental, la descripción y control de la documentación, y las búsquedas, apoyadas si se estima oportuno en el establecimiento de una lista controlada de descriptores.

Se ha logrado un programa sencillo, útil y completo que facilita la gestión y accesibilidad de los datos, e incluso la simplificación y racionalización de la producción documental, con lo que se posibilitará el aprovechamiento integral de los recursos de información documental administrativa.

\section{Palabras claves}

Archivos Administrativos / Informatización /Análisis Funcional/ Servicio Andaluz De Salud/ Archivo Central /

\section{Introducción}

La incorporación de los archivos administrativos al mundo de la informática o de las llamadas "nuevas" tecnologías de la información ha sido relativamente tardía; lo que resulta paradójico, ya que los archivos son una parte esencial dentro del ámbito de los centros y ciencias de la Información y Documentación.

A ello se añade el nivel de desarrollo alcanzado por las actuales tecnologías de la información y su grado de implantación en todos los sectores y ocupaciones diarias, incluidas especialmente las que tienen un reflejo documental en cualquier soporte.

Ambas razones hacen indispensable y urgente la incorporación inmediata de los archivos a las mencionadas tecnologías, ya que los archivos son los centros o unidades administrativas encargados de la ordenación y organización de la actividad de gestión documental en los organismos o instituciones en los que se adscriban.

Esta necesidad de incorporación a las nuevas tecnologías, mediante el desarrollo de un sistema integrado de gestión de las tareas del archivo, se hace apremiante e inaplazable cuando este archivo es de carácter central y, como es el caso del SAS, se inserta en una organización que genera y recibe una masa documental de gran volumen y complejidad tipológica.

Para lograr una correcta organización del trabajo técnico de un archivo administrativo de gestión o central es imprescindible disponer de una aplicación, de unos sistemas informáticos, que posibiliten tanto el desarrollo de la gestión interna del archivo como la relación archivística con las distintas unidades administrativas y usuarios de los servicios que presta dicho archivo central.

Por esta razón, y también por la falta en el mercado de un programa informático que cubriera completamente todos los requerimientos surgidos en la labor diaria del Archivo Central del Servicio Andaluz de Salud, después de analizados los disponibles en el momento, así como por la complejidad y dedicación necesarias para el trabajo informático, se decidió en su día la contratación, mediante concurso público, del desarrollo de un programa de gestión integral a medida.

Una vez adjudicado el concurso a la empresa Sadiel, S.A. se comenzaron los trabajos en 1998, centrándose tanto en el estudio y análisis del organismo Servicio Andaluz de Salud y su documentación, como en el desarrollo a medida del programa informático que posibilita la correcta gestión del Archivo Central del mismo.

Desde 1999 el programa llamado provisionalmente SIGAC (Sistema Informático de Gestión del Archivo Central) pasó a denominarse ACTA (Administración, Control y Tratamiento de Archivo) y a ser registrado como tal por parte del Servicio Andaluz de Salud. 
Todas las tareas para el desarrollo del programa se llevaron a cabo desde un primer momento en estrecha colaboración entre los componentes del equipo, informáticos y archiveros que se informaron mutuamente de las técnicas de cada uno de sus campos, en todas las reuniones de trabajo y en la labor diaria, guiándose siempre por una filosofía de conexión con los problemas cotidianos de un archivo administrativo. Todo ello para poder dar así una respuesta de la forma más racional y sencilla posible a las necesidades reales y cotidianas de cualquiera de éstos archivos.

En la metodología de trabajo seguida se ha primado el estudio del organismo y su documentación, lo que se concretó en el análisis de las funciones y los organigramas del Servicio Andaluz de Salud, la delimitación de las series documentales y la confección de una lista de términos controlados para la descripción y búsquedas documentales.

Además, como aproximación a las características generales del Archivo Central del SAS y de la documentación que custodia, se han recogido en una Guía los datos fundamentales de dicho archivo y unas recomendaciones o normas fundamentales de gestión (forma de hacer las transferencias al Archivo Central, organización de un archivo de oficina...)

\section{El Archivo Central del Servicio Andaluz de Salud}

La normativa por la que se formalizó la existencia del Archivo Central del SAS fue el decreto 233/1989 de 7 de noviembre (BOJA $n^{\circ}$ 94), que estableció el funcionamiento de los Archivos Centrales de la Consejerías, Organismos Autónomos y Empresas de la Junta de Andalucía, y su coordinación con el Archivo General de Andalucía. Con efectividad comenzó su marcha en diciembre de 1990, cuando se le dotó de personal por primera vez.

Sus funciones principales, como las de todo archivo de carácter central, son fundamentalmente:

- estudiar la organización y estructura del organismo, diacrónica y sincrónicamente, como marco referencial básico que tiene como producto la confección de organigramas,

- establecer los cuadros de clasificación y delimitar las series documentales,

- recoger y seleccionar anualmente la documentación producida y recibida por el organismo,

- organizar, describir y conservar esta documentación,

- facilitar el uso de los recursos documentales administrativos integrados en los fondos del archivo, como pruebas o antecedentes documentales y como información para la gestión administrativa y la toma de decisiones,
- velar por la adecuada organización y conservación de los documentos en las oficinas o unidades administrativas, mediante acciones de asesoramiento y formación sobre temas archivísticos o de gestión documental: organización de archivos, articulación de circuitos documentales...

- estudiar la racionalización y normalización de dichos circuitos documentales, como resultado de los procesos de trabajo en el organismo,

- asesorar a los centros administrativos que dependan del organismo, en el caso del SAS los centros administrativos sanitarios, en las mencionadas cuestiones archivísticas o de gestión documental,

- realizar las transferencias al Archivo General de Andalucía, como archivo de carácter intermedio dentro de la red de centros del sistema andaluz de archivos,

- colaborar con otros organismos, instituciones y profesionales, en materias técnicas archivísticas,

- sin olvidar llevar a cabo todas estas las actividades para la realización de las mencionadas funciones, de forma que sean conducentes a la correcta y adecuada organización, control y servicio de la documentación custodiada en el archivo.

La carga de trabajo que supone el cumplimento de todas estas funciones se ve aumentada cuando el archivo central forma parte y sirve a un organismo de las dimensiones, complejidad y trascendencia del Servicio Andaluz de Salud. Los servicios centrales de este organismo, que gestiona un presupuesto de miles de millones de pesetas y cuenta con más de setenta y cuatro mil trabajadores en sus distintos centros dependientes, generan evidentemente una gran cantidad y diversidad de procedimientos y circuitos de trabajo, que tienen su correspondiente reflejo documental. Y además, dichos servicios centrales del SAS reciben una enorme cantidad de documentos que se integran también en los grupos o series documentales.

Es evidente así, que la complejidad, cantidad y trascendencia de la documentación generada y recibida por el Servicio Andaluz de Salud, y por tanto organizada y conservada por su Archivo Central, es enorme. Los cincuenta y tres órganos de dirección y unidades administrativas tienen delimitadas más de mil ochocientas series o grupos documentales. Además en los depósitos del archivo se custodian unas 26.000 unidades de instalación, y la previsión anual de crecimiento medio anual es de dos mil quinientas de éstas unidades.

Por supuesto, como ya hemos señalado, un archivo central de éstas características necesitaba disponer de forma urgente, ya desde el inicio de su andadura, de unas herramientas informáticas que facilitaran su labor. Y ya en ese primer momento se vió la necesidad de que el programa informático que se eligiera o desarrollara abarcara toda la gestión de las tareas del archivo central de una forma integral, desde la caracteriza- 
ción de series documentales, a la realización de búsquedas o facilitación de acceso a la documentación, pasando por los trámites de remisiones o transferencias, todo ello citado a modo de ejemplo.

Pasaremos seguidamente a describir de una forma más detallada la labores previas archivísticas e informáticas en las que se basa el ACTA, la estructura o módulos del mismo, y las líneas de trabajo en desarrollo y proyecto.

\section{Estudios previos y desarrollo de la informatización}

Como hemos comentado, la filosofía que inspiró el método de trabajo seguido para el desarrollo del ACTA ha sido la colaboración entre todos los miembros del equipo multidisciplinar que componíamos con un objetivo básico: conseguir que el personal informático tuviera conciencia de las características y peculiaridades que entraña el trabajo técnico de archivo, y que los documentalistas y archiveros se acercasen a su vez a la terminología y procedimiento de trabajo de los informáticos.

En primer lugar, y como fase fundamental para iniciar el trabajo de informatización se llevó a cabo el análisis funcional de las actividades y tareas propias del Archivo Central incluyendo el estudio de los procesos y circuitos de trabajo en el mismo y de los documentos generados por las mencionadas tareas.

En segundo lugar, se realizó el estudio funcional de la documentación del SAS y del marco orgánico de la misma: se analizó la evolución estructural del SAS desde su creación hasta nuestros días, identificando las unidades administrativas existentes en cada uno de los trece organigramas que reflejan la estructura orgánica del SAS y las funciones asignadas a cada una de ellas. Sobre la base de esos datos recopilados se procedió a elaborar los correspondientes cuadros orgánicos y funcionales, y una lista inicial de términos de descripción de la documentación, similares a los índices y listas de autoridad tradicionalmente utilizados en los archivos.

Seguidamente se desarrollaron e instalaron los primeros módulos de la aplicación informática, el de administrador (o base estructural) y el de usuario (o de trabajo de gestión del archivo), ya que se optó como lo más operativo en separar las tareas archivísticas de gestión diaria (remisiones, descripción, préstamos...) de las que implicasen una definición o cambio en la estructura organizativa en cuanto a la base orgánico funcional, la disponibilidad de depósitos e instalación y el control de los términos descriptivos. Paralelamente, y en colaboración con el personal informático del equipo, se fue sometiendo el programa a un chequeo continuo con objeto de poder apreciar los resultados que ofrecía e incorporar posibles mejoras.

Se logró tras todo este proceso, el desarrollo completo de los módulos administrador y usuario del programa y además una serie de productos documentales, que son a su vez instrumentos de trabajo: el estudio estructural con sus cuadros de clasificación y repertorio de series documentales, una lista controlada de términos de descripción, la guía del Archivo Central, y la puesta al día de la compilación de normativa interna del SAS.

El estudio de la evolución estructural organizativa del SAS a nivel orgánico y funcional fue plasmado en la confección por cada unidad administrativa de los correspondientes cuadros de clasificación, tras un proceso de identificación, normalización terminológica y codificación de sus series documentales. Este trabajo se basó en entrevistas con el personal de cada órgano de dirección y unidad administrativa, y en el estudio de las normas legales y de la propia documentación mediante unas "fichas de identificación" que constituyen el repertorio de series.

Además se elaboró una lista de control de términos administrativo-sanitarios, llamada "diccionario" en el programa, como base para la descripción documental y la posterior búsqueda y recuperación de documentos a los que se quiere acceder. En la actualidad se sigue trabajando en ella, incorporando términos de la documentación del SAS y de fuentes externas, y se ha comenzado a estructurarlos como descriptores o materiales para un futuro tesauro.

Por último, se realizó la guía del Archivo Central del SAS, que figura como una opción del sistema informático desarrollado, y como producto del trabajo del análisis funcional del organismo se realizó la puesta al día de la "compilación normativa" que recoge las referencias de normativa interna de dicho organismo desde el año 1995

\section{Descripción del Sistema}

El programa ACTA consta de tres módulos que agrupan las actividades realizadas para el funcionamiento del sistema: el módulo del Administrador del Sistema, el módulo de Usuario, y el módulo de Oficinas

Con el módulo del Administrador del Sistema se realizan todas aquellas funciones que permiten la definición del mismo: altas y bajas de usuarios del sistema, claves de acceso, estructura del archivo, organigramas, términos de descripción, etc. Podría denominarse también como módulo de estructura, ya que define la estructura lógica y física del archivo.

En el módulo de Usuario los archiveros usuarios de la aplicación gestionan las actividades propias de un archivo, es decir, préstamos, accesos, transferencias, remisiones, expurgos, gestión de espacio, descripción de documentos, etc.

El módulo de Oficinas posibilita la conexión de los archivos de oficina o gestión de las distintas unidades administrativas con el Archivo Central, concretamente en las tareas de transferencia documental y en la gestión del acceso a los documentos de archivo ya remitidos al Archivo Central. 


\section{I. Módulo de Administrador del Sistema}

El primer paso a seguir antes de entrar en este módulo es identificarse como administrador, debiendo introducir el identificador y clave de acceso, evitando así que personas no autorizadas puedan manipular las herramientas de trabajo y gestión del Archivo Central.

Una vez dentro de la aplicación, se presenta un menú con las funciones en forma de iconos.

\section{I.I. Organigramas}

En ésta función se define el organigrama general del archivo, especificando cuál es la estructura del fondo documental y aportando datos esenciales de forma gráfica y jerárquica como son los órganos productores (unidades administrativas) y la denominación de las series o grupos documentales. Hemos preferido hablar de "grupos documentales", ya que muchas veces necesitamos identificar y tratar agrupaciones documentales que no son propiamente series, desde el estricto punto de vista técnico archivístico.

Por otra parte, no sólo existe la posibilidad de visualizar el organigrama actual, sino que se tiene acceso a todos los anteriores y se puede consultar su fecha de creación, fecha de baja, publicación como norma legal, posibles observaciones, etc.

Cuando se crea un nuevo organigrama existe un módulo de generación de organigramas, con el que no es necesario construir el organigrama desde cero sino que podemos basarnos en el anterior y establecer las modificaciones oportunas: nuevas unidades administrativas, divisiones o uniones entre ellas, desapariciones, etc., teniendo en cuenta que si queremos cada unidad administrativa "llevará" consigo todos sus grupos documentales o series a su nueva situación en el organigrama que estamos construyendo.

En el organigrama, se pueden crear nuevos grupos documentales, cambiarlos de unidades administrativas o cerrarlos en cuyo caso aparecen con un icono que indica esta circunstancia.

Se integra en este módulo el control de fichas de series y/o grupos documentales, en el que se visualizan las fichas de identificación y valoración de las series y/o grupos que están estudiados y se tiene opción de impresión de las mismas.

\section{I.2. Estructura}

Con esta función se define la estructura física del depósito o de los distintos depósitos del archivo, de forma tal que se indicará los archivos físicos que se gestionan, número de estanterías por archivo, baldas por estantería y cuantas cajas tienen cabida en cada balda.

\section{I.3. Diccionario}

El diccionario agrupa los descriptores o términos de descripción documental que ayudan a hacer accesibles los documentos y su información a los interesados. Se pueden definir sinónimos y otras relaciones de descriptores para facilitar aún más la búsqueda de un documento, o limitarse a una simple lista de términos o a un índice.

Al no existir un tesauro de términos administrativo-sanitarios pensado y válido para el trabajo en archivos, los términos recogidos en el diccionario constituyen unos materiales para su elaboración totalmente novedosos, y son además una herramienta de trabajo fundamental para normalizar y unificar criterios en el desarrollo del mencionado trabajo de archivo.

Como material complementario se ha confeccionado una "Guía para la recuperación de la Información" que se implementa como manual de procedimiento interno de trabajo que facilite las consultas o búsquedas en el ACTA, y se incorporará al texto de la opción "Guía".
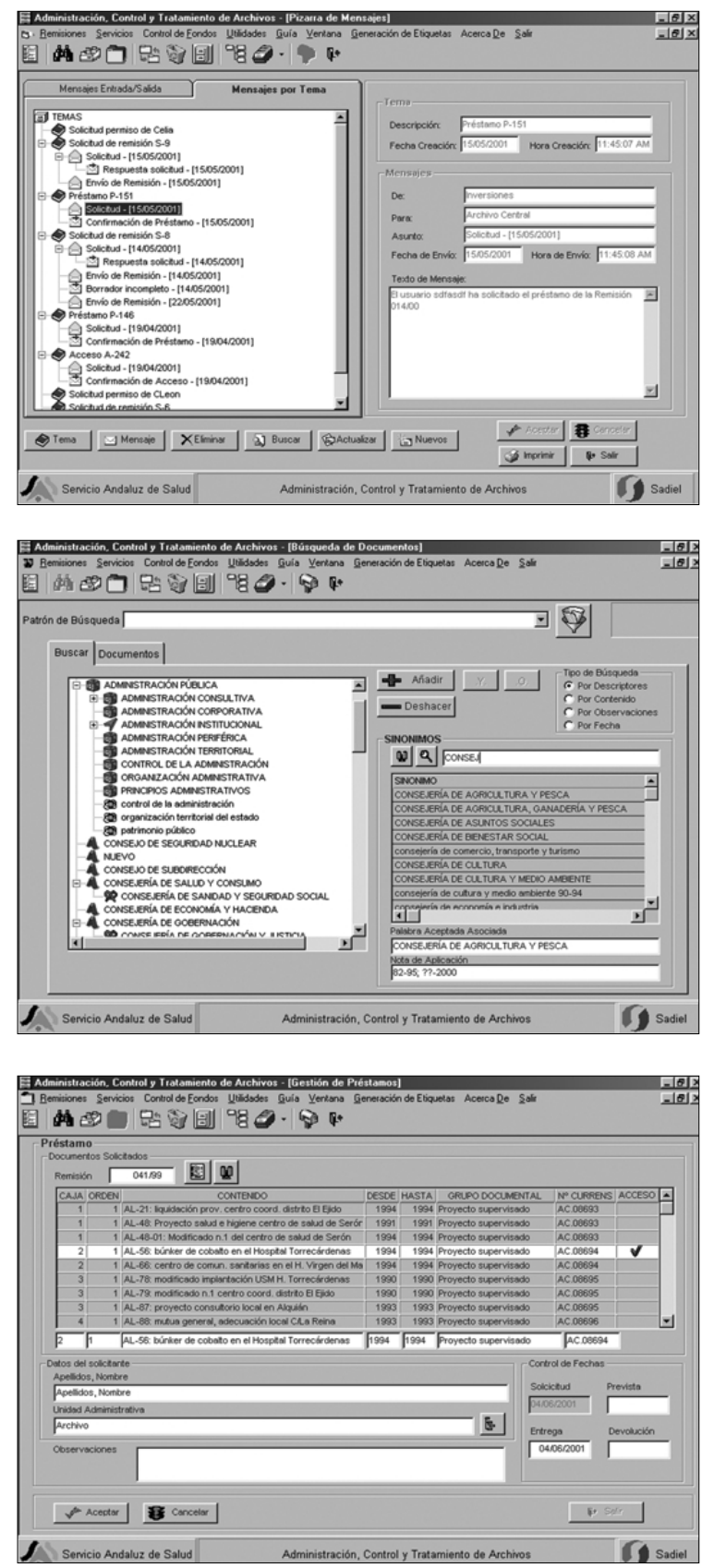


\section{I.4. Imprimir}

Se permite la obtención de distintos listados dependiendo de la información visualizada en ese momento; en el manejo del ACTA no es necesario imprimir para "ver los listados", pues en todo momento se visualizan en pantalla.

En esta opción también pueden imprimirse las etiquetas de las cajas con su correspondiente signatura.

\section{I.5. Estadísticas}

Esta opción permite obtener las estadísticas definidas para el Archivo que servirán para la confección de la memoria de gestión anual del Archivo Central.
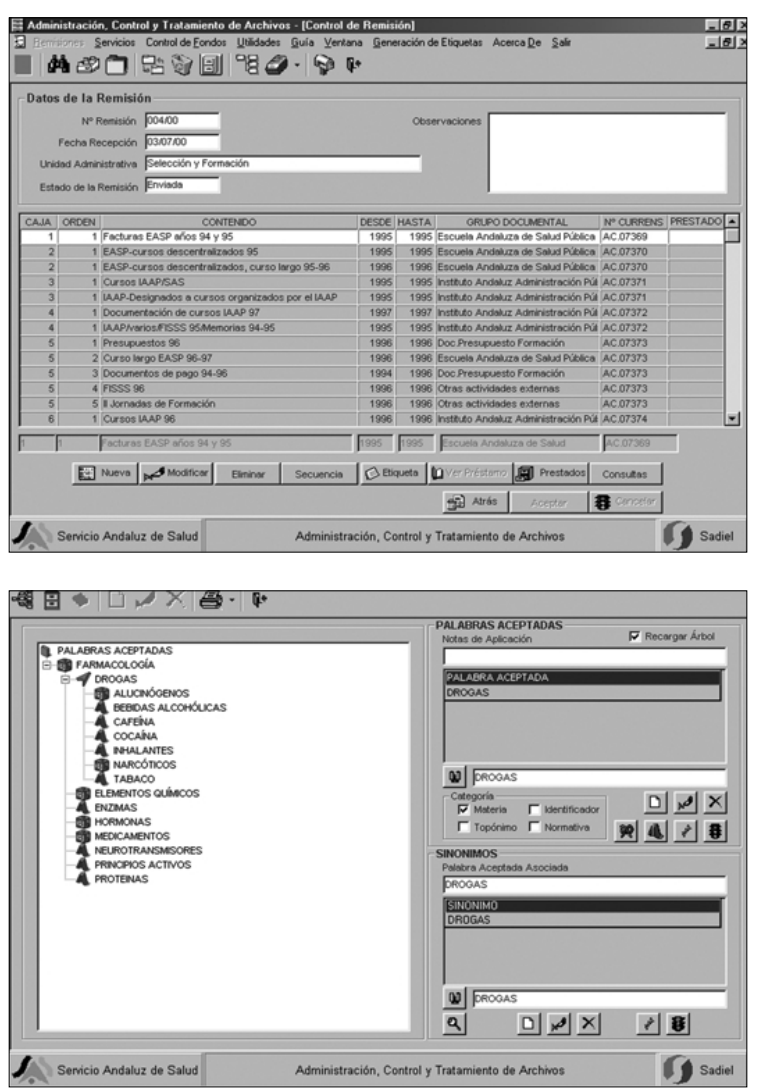

\section{I.6. Guía}

En la guía se visualiza una serie de paginas HTML que da información de los datos más significativos del Archivo Central: ubicación, funciones, cuadros de clasificación, etc., además de recomendaciones de organización para los archivos de oficina (por ejemplo gestión de remisiones) y de utilización del ACTA (recuperación de información, etc.)

\subsection{Módulo de Usuario Final}

Este módulo es el que utilizarán los técnicos que trabajan diariamente en el Archivo Central una vez autorizados como usuarios del sistema por el Administra- dor del mismo. El primer paso a seguir antes de entrar en este módulo es identificarse como usuario, debiendo introducir el identificador y clave de acceso.

Una vez dentro de la aplicación, se presenta un menú con las funciones mas frecuentes en forma de iconos

\subsection{Remisiones}

Cada remisión o transferencia supone la organización, descripción y traslado de un conjunto de documentos desde las unidades administrativas al Archivo Central para su custodia. Para que esto sea posible es necesario cumplir una tramitación administrativa, formalizada a través de una hojas normalizadas ("hojas de remisión") en las que se expresan una serie de datos referidos a la documentación y al hecho mismo de la remisión desde el archivo de gestión. En este módulo es posible llevar un exhaustivo control de la documentación y llegar a describirla mediante una serie de palabras clave o descriptores lo que facilitará su posterior localización. Algunas de las funcionalidades de la opción son:

- Localización de documentos: para controlar los documentos remitidos.

- Control de Fechas: para tener constancia del año al que pertenece la remisión

- Unidad administrativa que lo realiza: quién realiza la remisión.

- Estado: si la remisión está pendiente de revisión, de formalización (enviada a la oficina para su firma) o ya formalizada (cerrada).

- Impresión del Acta de Remisiones

- Listados de Remisiones: informes de las remisiones recibidas con la posibilidad de filtrado por fecha, unidad administrativa y estado.

- Introducción de descriptores: entorno visual para la consulta/incorporación de descriptores o términos.

\subsubsection{Búsquedas}

Herramienta muy útil a la hora de localizar cualquier tipo de documento. Se realizan búsquedas a través de una o varias palabras clave o descriptores, e incluso por el texto del contenido de la descripción y el campo de observaciones, se pueden acotar cronológicamente y utilizar los operadores "y" u "o" aisladamente o en conjunto. Se obtiene una relación de documentos coincidentes, detallando en cada uno de ellos el número de la remisión y la unidad administrativa a la que pertenece, así como una breve descripción.

Se ha desarrollado una optimización de las búsquedas documentales mediante entorno visual, y se ha llevado a cabo un procedimiento almacenado que actualiza diariamente la tabla de descriptores o términos asociados a cada documento ("recalcula").

\subsubsection{Accesos}

Con esta opción se gestiona las consultas que realizan los usuarios a documentos del archivo. El sistema controla los documentos, usuarios que lo solicitan, fecha, 
tipo de copia que se lleva, emisión de impresos de solicitud de acceso al archivo, etc.

\subsubsection{Préstamos}

Con esta función se gestionan los Préstamos, es decir documentos que han solicitado los usuarios para su consulta y que todavía tienen en su poder, permitiendo saber quien tiene cada documento y la fecha prevista de devolución. Además dispone de un control de documentos vencidos, pudiendo saber que documentos deberían haber sido devueltos a una fecha determinada, y se ha llegado a individualizar los préstamos por expedientes o unidades documentales.

\subsubsection{Transferencias}

Igualmente se gestionan las transferencias de grupos documentales desde el Archivo Central del SAS al AGA (Archivo General de Andalucía), dejando constancia de dicha operación para poder ser consultada a posteriori. Mediante esta opción de menú es posible controlar:

- El estado de tramitación de cualquier transferencia.

- Documentos que se transfieren

- Unidad administrativa que realizó la remisión al Archivo Central

- Grupos o series documentales transferidas

- Fechas de los documentos transferidos.

- Etc.

\subsubsection{Expurgos}

En sentido estricto es la eliminación de documentos que no interesa conservar. Para la técnica archivística es la tarea que consiste en la selección y posterior destrucción de los documentos cuyos valores intrínsecos se consideren ya desaparecidos o inexistentes tras su valoración y selección.

Las funciones principales de ésta opción son:

- Impresión del acta de expurgo

- Impresión del contenido del expurgo

- Control del estado del expurgo (cerrado, tramitado, pendiente)

- Tramitaciones

- Consultas

- Etc.

\subsubsection{Gestión del espacio}

Con esta opción se obtiene información de la ocupación del archivo: número de cajas totales, número de cajas ocupadas y porcentaje de ocupación de cada archivo, estantería o balda con un simple "click" de ratón, y puede elegirse el lugar para ubicar una remisión de documentación.

Otra funcionalidad asociada es la posibilidad de ver qué documentos tiene una determinada caja y cuál es el contenido de cada uno de ellos; lo que además posibilita de revisión de los fondos topográficamente
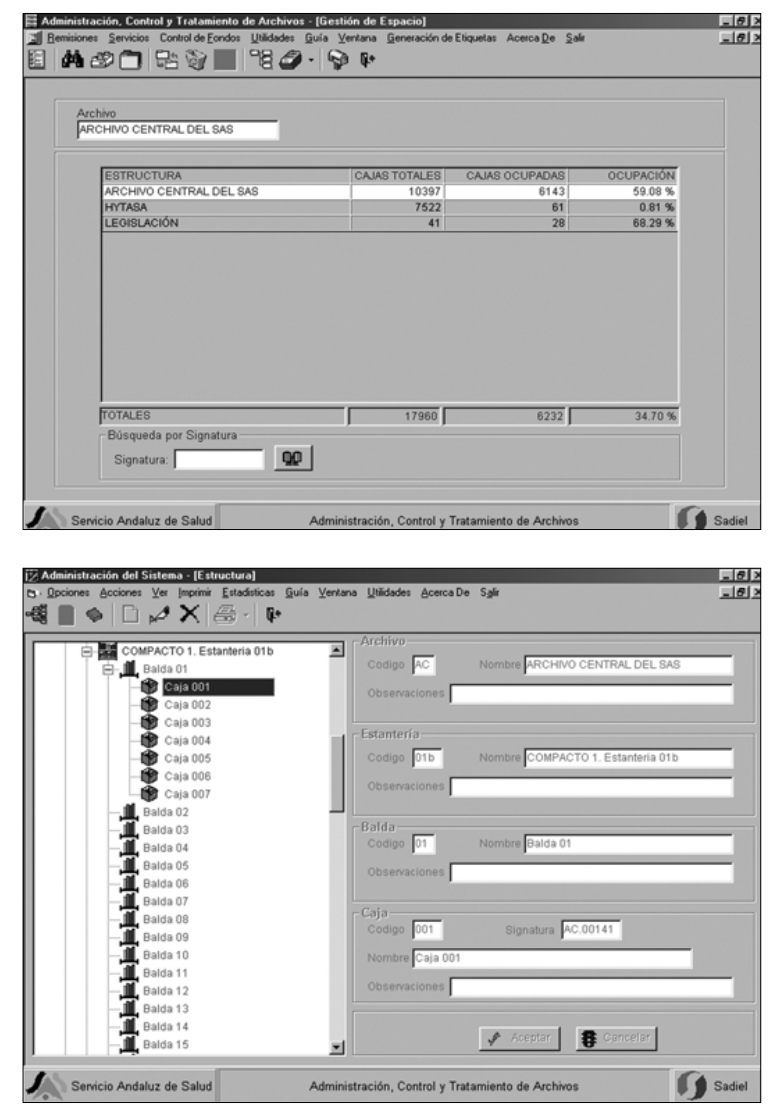

\subsubsection{Cuadro de Clasificación}

Muestra de forma gráfica (en forma de árbol) cuál es el cuadro orgánico-funcional que estructura el fondo documental, desplegando cada unidad administrativa con sus series o grupos documentales.

Es posible visualizar e imprimir el cuadro de clasificación completo, o sólo la parte correspondiente a una serie o grupo documental (lo que posibilita el inventario por series documentales). Al imprimir el cuadro de clasificación disponemos además de los dígitos de cada serie.

\subsubsection{Inventario}

Con esta función es posible realizar la impresión de los inventarios correspondientes a varios criterios: topográfico de cada depósito del archivo, por cuadro de

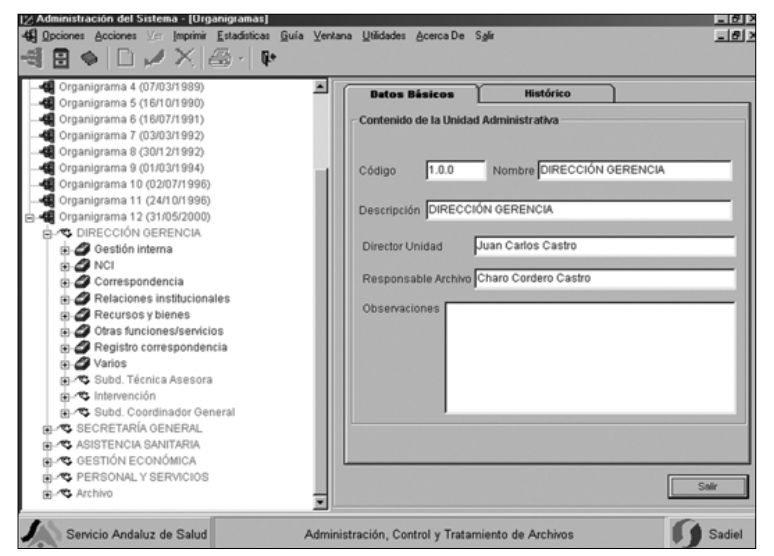




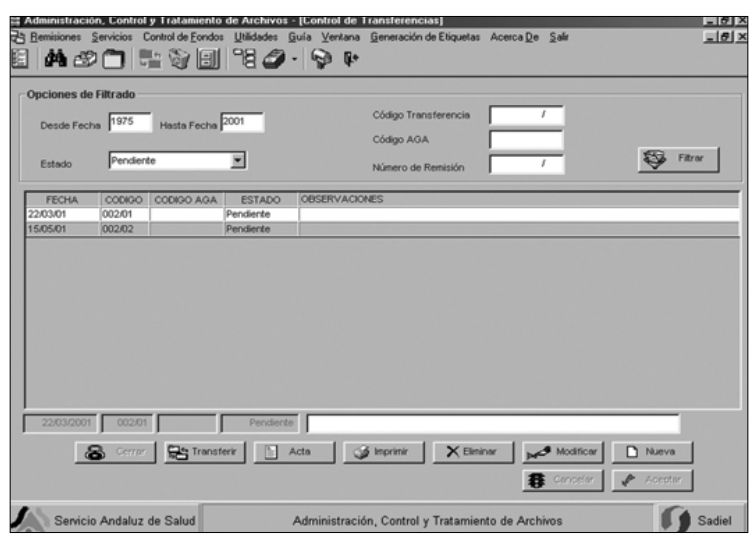

clasificación de cada unidad administrativa, o por serie o grupo documental determinado.

\subsection{Mensajes}

Por último, esta opción facilita la conexión con los archivos de oficina o con las unidades administrativas, en cuanto a la recepción y envío de mensajes con los mismos.

\subsection{Módulo de oficinas}

En la actualidad la optimización de los recursos del ACTA ha hecho posible el desarrollo del módulo de oficinas para la conexión de los archivos de oficina o gestión de cada unidad administrativa con el Archivo Central: se basa en dos puntos facilitar y automatizar tanto las remisiones de documentos como las búsquedas desde las oficinas, con una mínima necesidad de formación en el uso del módulo por parte del personal de las oficinas.

\subsection{Remisiones desde las oficinas}

La primera opción que contiene es la de remisiones: su finalidad es facilitar a la oficina la realización del borrador de la remisión, su estructura es similar a la del módulo de usuario, con las diferencias de que se accede sólo al cuadro de clasificación de la oficina que realiza la remisión y no se puede asignar signatura de Archivo Central.

\subsubsection{Búsquedas}

La opción de búsqueda también es similar a la que contiene el módulo de usuario, aunque por defecto sólo puede realizar búsquedas sobre la documentación que la propia oficina ha remitido al Archivo Central; está previsto que éste pueda autorizar otros niveles de acceso en casos puntuales como series horizontales o series correspondientes a funciones asumidas posteriormente por una unidad administrativa distinta a la que remitió en su día la documentación.

\subsubsection{Mensajes}

El módulo de oficinas cuenta también con una opción de mensajes para intercambiar los mismos con el Ar- chivo Central, con objeto de simplificar la relación cotidiana con el mismo.

\section{Resultados y líneas de trabajo}

Como fruto de las tareas y la experiencia desarrollada hemos obtenido una serie de resultados y conclusiones y se han perfilado para un futuro varias líneas de trabajo, algunas ya en pleno desarrollo:

- Ha sido esencial, para el éxito de los trabajos técnicos del sistema, la colaboración continua entre archiveros e informáticos, informándose tanto de los temas técnicos respectivos como de las dificultades encontradas y de las correcciones oportunas efectuadas. Sin esta colaboración continua el programa no habría resultado útil o simplemente no habría existido.

- Se ha posibilitado enormemente la agilización de todas las tareas técnicas archivísticas del Archivo Central, en cuanto a gestión integral de las mismas y control de los fondos. Se han agilizado las tareas de descripción documental, los trabajos de delimitación y caracterización de series o grupos documentales y los estudios de valoración y selección documental, y se ha facilitado el acceso a la documentación custodiada en el Archivo Central.

- Se está trabajando en una serie de manuales de recomendaciones de organización de archivos y de utilización del ACTA, pretendiendo llegar a la confección de un manual de gestión documental que recopile todas las normas, circuitos y procesos que informan cada serie documental en el Servicio Andaluz de Salud.

- Se puede llegar por último a un plan integral de información y gestión documental, que englobe al ACTA y a los procesos documentales desarrollados en todas las unidades administrativas, especialmente las más directamente implicadas en dichos procesos documentales o en general de carácter informativo. Dicho plan supondrá, mediante la racionalización de la producción documental y la simplificación de sus correspondientes procedimientos, el aprovechamiento y control total e integral de los recursos de información documental administrativa del Servicio Andaluz de Salud.

Con el ACTA, programa especialmente desarrollado para el Archivo Central del Servicio Andaluz de Salud, dicho organismo dispone de una herramienta de trabajo de fácil uso que puede implementarse en cualquier archivo administrativo, que resuelve de forma completa todas las necesidades de gestión de los mismos, y que ha sido desarrollada con óptimos resultados gracias a la realización por el propio archivo del oportuno análisis funcional y procedimental, y a la colaboración continua entre archiveros e informáticos. 